\title{
Autologous transplantation of adipose-derived mesenchymal stem cells ameliorates streptozotocin-induced diabetic nephropathy in rats by inhibiting oxidative stress, pro-inflammatory cytokines and the p38 MAPK signaling pathway
}

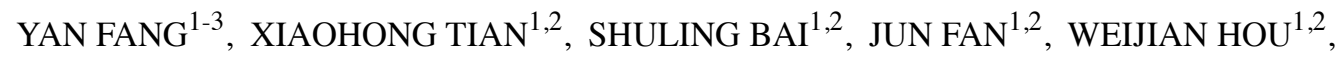

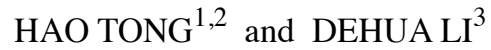 \\ ${ }^{1}$ Department of Anatomy and ${ }^{2}$ Tissue Engineering, College of Basic Medical Sciences, China Medical University, \\ Shenyang 110001; ${ }^{3}$ Department of Anatomy, Liaoning Medical University, Jinzhou 121001, \\ Liaoning Province, P.R. China
}

Received February 1, 2012; Accepted April 2, 2012

DOI: $10.3892 / \mathrm{ijmm} .2012 .977$

\begin{abstract}
Diabetic nephropathy is the leading cause of endstage renal disease. The aim of this study was to investigate the renoprotective effects of autologous transplantation of adiposederived mesenchymal stem cells (ADMSCs) and to delineate its underlying mechanisms of action in diabetic nephropathy. Diabetes was induced in adult male Sprague-Dawley rats by streptozotocin (STZ) injection. ADMSCs were administered intravenously 4 weeks after STZ injection and metabolic indices and renal structure were assessed (12 weeks). Markers of diabetes including blood glucose, cholesterol, triglycerides, urea nitrogen and creatinine were measured. Renal pathology, levels of oxidative stress and the expression of pro-inflammatory cytokines and the MAPK signaling pathway members were also determined. Autologous transplantation of ADMSCs significantly attenuated common metabolic disorder symptoms associated with diabetes. Furthermore, ADMSC administration minimized pathological alterations, reduced oxidative damage and suppressed the expression of pro-inflammatory cytokines in the renal tissues of diabetic rats. ADMSC transplantation also decreased the expression of p-p38, p-ERK and p-JNK, which are all important molecules of the MAPK signaling pathway. In conclusion, we provide experimental evidence demonstrating that autologous transplantation of ADMSCs can be used therapeutically to improve metabolic disorder and relieve renal damage induced by diabetes, and that the
\end{abstract}

Correspondence to: Dr Shuling Bai, Department of Anatomy, Department of Tissue Engineering, College of Basic Medical Sciences, China Medical University, 92 North Second Road, Shenyang 110001, Liaoning Province, P.R. China

E-mail: shuling_bai@163.com

Key words: adipose-derived mesenchymal stem cells, diabetic nephropathy, oxidative stress, pro-inflammatory cytokines, p38 MAPK signaling pathway key mechanisms underlying the positive therapeutic impact of ADMSC treatment in kidneys could be due to the suppression of inflammatory response and oxidative stress.

\section{Introduction}

Diabetic nephropathy is a major microvascular complication in patients with diabetes and remains the leading cause of chronic kidney disease, accounting for approximately a half of all end-stage renal disease worldwide $(1,2)$. The key pathologic features of diabetic nephropathy include microalbuminurea, mesangial cell (MC) hypertrophy, thickened glomerular and tubular basement membrane, tubulointerstitial fibrosis, and low grade of renal inflammation. It is now well recognized that the pathogenesis of diabetic nephropathy is multifactorial. Over the last decade, diverse pathological mechanisms have been proposed to be involved in the onset and development of diabetic nephropathy, such as genetic and hemodynamic factors, oxidative stress and cytokine signaling $(3,4)$. Nowadays, therapies for diabetic nephropathy have been limited to drugs that improve blood pressure or control blood glucose levels. However, these therapies are not very effective in blocking renal damage and co-treatment with renoprotective drugs often leads to toxicity and reduction in efficacy $(5,6)$. There is thus an imperative need to develop effective therapeutic strategies to preserve normal renal function or to halt the progression of diabetic nephropathy.

Mesenchymal stem cells (MSCs) are multipotential nonhematopoietic progenitor cells that can differentiate into a variety of cell types, including osteoblasts, chondrocytes and adipocytes $(7,8)$. Accumulating evidence has shown that MSC-based therapy is of noteworthy potential in treating various human diseases (9). Bone marrow-derived MSCs (BMMSCs) have long been considered to be the standard for stem cell sources in cell-based therapy and tissue engineering. However, the use of BMMSCs is still problematic because of the low number of MSCs found in bone marrow aspirates and the invasive procedure associated with obtaining them. When 
compared to BMMSCs, adipose-derived MSCs (ADMSCs) are equally capable of differentiating into cells and tissues of mesodermal origin. In addition, they offer some advantages as an attractive, readily available adult stem cell because of the ease of harvest and their abundance (10). Based on these, adipose tissue may represent a source of stem cells that could have far-reaching effects on several fields. More recently, a number of experimental studies using animal models of kidney disease have clearly demonstrated that MSC-based therapy is capable of preserving renal parenchymal integrity from acute ischemic injury and improving kidney function from acute damage (11-15). Additionally, MSC-based therapies have been considered to be a promising new means of treating diabetes and its associated complications (16). For instance, Vanikar et al (17) reported that co-transplantation of adipose tissuederived insulin-secreting MSCs and hematopoietic stem cells is beneficial in reducing exogenous insulin requirement in patients with insulin-dependent diabetes mellitus. It has also been demonstrated that transplantation of ADMSCs attenuates diabetic bladder dysfunction and diabetic retinopathy in a diabetic rat model $(18,19)$.

Based on these previous findings, we therefore raised the hypothesis that cytotherapy using autologous ADMSC transplantation is beneficial in alleviating diabetic nephropathy. Accordingly, in the present study, we investigated the therapeutic potential of autologous ADMSC transplantation in delaying the progression of diabetic nephropathy using a streptozotocin (STZ)-induced diabetic rat model.

\section{Materials and methods}

Animals and ethics. Eight-week-old male Sprague-Dawley rats weighing 200-250 g were purchased from the Experimental Animal Centre of China Medical University. They were housed in plastic cages under controlled environmental conditions $\left(23 \pm 2^{\circ} \mathrm{C}\right.$ and a 12 -h light/dark cycle) and had free access to food and water unless otherwise stated. All experimental animal procedures were approved by the Institute of Animal Care and Use Committee at China Medical University and performed in accordance to the Guide for the Care and Use of Laboratory Animals.

Experimental groups and isolation of ADMSCs. Thirty-two rats were randomized into the following four groups (n=8/group): i) the non-diabetic group, in which rats received intraperitoneal (i.p.) injection of citrate buffer alone, ii) the ADMSC group, in which STZ-induced diabetic rats received transplantation of ADMSCs, iii) the vehicle group, in which STZ-induced diabetic rats treated with culture medium and iv) the diabetic group, STZ-induced diabetic rats without any further treatment.

ADMSCs were isolated from adipose tissue of the rats in the ADMSC group according to the method developed by Zuk et al (20) with minor modifications. In brief, the rats were anesthetized with $10 \%$ chloral hydrate $(350 \mathrm{mg} / \mathrm{kg}$, i.p.) 14 days before ADMSCs transplantation and the inguinal subcutaneous adipose tissue was collected under sterile conditions from these rats. Harvested tissue was washed with sterile phosphate-buffered saline (PBS), minced into small pieces and enzymatically dissociated with $0.1 \%$ type I collagenase (Sigma-Aldrich, St. Louis, MO, USA) solution at $37^{\circ} \mathrm{C}$ in a water-bath shaker for $30 \mathrm{~min}$ with gentle agitation. Type I collagenase was inactivated with an equal volume of Dulbecco's Modified Eagle's Medium (DMEM) (Hyclone Laboratories, Inc., Logan, UT, USA) supplemented with $10 \%$ fetal bovine serum (FBS) (Hyclone Laboratories). Digested adipose tissue was filtered twice through a $100-\mu \mathrm{m}$ and a $25-\mu \mathrm{m}$ nylon membrane to eliminate the undigested fragments. Multipotent ADMSCs were harvested by centrifugation at $1,000 \mathrm{x} g$ for $10 \mathrm{~min}$ at room temperature. The cell pellet thus obtained was resuspended in DMEM containing 10\% FBS and incubated for $24 \mathrm{~h}$ at $37^{\circ} \mathrm{C}$ in $5 \% \mathrm{CO}_{2}$. Non-adherent cells were removed after $72 \mathrm{~h}$ and adherent cells were passed at low density into new flasks. When the cells reached $80 \%$ confluence, adherent cells were digested with $0.125 \%$ trypsin and $0.01 \%$ EDTA and then replated at a 1:3 dilution.

Verification of ADMSCs. The isolated ADMSC phenotype was confirmed by assessing their surface markers using flow cytometric analysis with appropriate fluorescein isothiocyanate (FITC)-conjugated antibodies. In brief, the harvested second-passage ADMSCs were washed twice with cold PBS solution, suspended in blocking buffer and incubated for 30 min at $4^{\circ} \mathrm{C}$ in the dark with the FITC-conjugated antibodies against CD29 (BD Biosciences, San Jose, CA, USA), CD34 (Novus Biologicals, Littleton, CO, USA), CD90 (Invitrogen Life Technologies, Carlsbad, CA, USA), CD31 (Santa Cruz Biotechnology, Inc., Santa Cruz, CA, USA), CD45 (BD Biosciences) and CD13 (BioLegend, San Diego, CA, USA). After being washed twice with PBS, ADMSCs were suspended in PBS and analyzed by FACSCalibur flow cytometry using CellQuest software (BD Biosciences).

ADMSCs labeling for in vivo tracking. Thirty minutes prior to autologous transplantatting ADMSCs, the Vybrant CM-Dil cell-labeling solution (50 $\mu \mathrm{g} / \mathrm{ml}$; Invitrogen Life Technologies) was added to the culture medium for ADMSCs labeling. The labeled cells were analyzed under a fluorescence microscope.

Induction of experimental diabetes and autologous ADMSCs transplantation. Rats were injected intraperitoneally with $40 \mathrm{mg} / \mathrm{kg}$ body weight of STZ (Sigma) prepared in $0.1 \mathrm{ml}$ citrate buffer $(\mathrm{pH} 4.5)$ for 5 consecutive days to induce type 1 diabetes or with $0.1 \mathrm{ml}$ citrate buffer as non-diabetic normal control. Two weeks following STZ injection, fasting glucose levels (4-h fast) were measured, and the experimental rats with symptoms such as polyphagia, polydipsia and polyuria together with a blood glucose level above $300 \mathrm{mg} / \mathrm{dl}$ were considered to be diabetic. For the rats in the ADMSC group, intravenous (i.v.) infusion of autologous ADMSCs $\left(1.0 \times 10^{7}\right)$ was performed 4 weeks after the onset diabetes via the tail vein. Animals in the vehicle group received an equal volume of culture medium at the same time. At 12 weeks, all animals were anesthetized and blood samples were drawn from the orbital venous plexus. Blood samples were anticoagulated with EDTA and centrifuged at $300 \mathrm{x}$ g for $5 \mathrm{~min}$ at $4^{\circ} \mathrm{C}$ and the serum was stored at $-20^{\circ} \mathrm{C}$ until analysis. All rats were then sacrificed by decapitation and their kidneys were rapidly excised, washed with PBS and divided into two pieces, one frozen in liquid nitrogen for western blot analysis and the remaining part fixed in $10 \%$ formalin solution for histopathological study. 
Biochemical analysis. The blood samples from experimental animals were obtained following by overnight fasting. Plasma glucose and blood urea nitrogen (BUN) levels were determined using commercially available kits (Jiancheng Bioengineering Institute, Nanjing, China). Plasma insulin was measured with a radioimmunoassay (RIA) kit (Atom High-Tech Co., Ltd., Beijing, China). Plasma cholesterol, triglyceride and creatinine levels were estimated spectrophotometrically using a commercial assay kit (BioSystems, Barcelona, Spain).

Oxidative stress and analysis of pro-inflammatory cytokines. Malondialdehyde (MDA) levels in the renal tissues, as an indicator of lipid peroxidation, were determined using a commercial kit (Jiancheng Bioengineering Institute). The levels of protein carbonyl group were measured by the 2, 4-dinitrophenylhydrazine (DNPH) method as described previously (21). Pro-inflammatory cytokines in the renal tissues, including tumor necrosis factor (TNF)- $\alpha$, interleukin (IL)-1 $\beta$ and IL-6, were determined with ELISA kits from R\&D Systems (Minneapolis, MN, USA). These measurements were performed according to the respective manufacturer's instructions.

Renal morphology assessment. Periodic acid-Schiff (PAS) stain was performed to assess tubular integrity and renal morphology as previously described. Briefly, the kidneys were fixed in $10 \%$ neutral-buffered formaldehyde, dehydrated through a gradual series of alcohol, embedded in paraffin and sectioned at 4- $\mu \mathrm{m}$ thickness. Paraffin kidney sections were then stained with hematoxylin and periodic acid-Schiff reagents for glomerular histology. After dehydration, the histopathological changes of stained glomeruli were examined by well trained pathologists under a light microscope.

Western blot analysis. Frozen renal tissues were homogenized in ice-cold radioimmunoprecipitation (RIPA) lysis buffer (Beyotime Institute of Biotechnology, Haimen, China). After centrifugation $\left(12,000 \mathrm{x} \mathrm{g}, 10 \mathrm{~min}\right.$ at $\left.4^{\circ} \mathrm{C}\right)$, the supernatant was collected and protein concentrations were determined by using a bovine serum albumin (BSA) standard line. Equal amounts of proteins were separated on sodium dodecyl sulfate polyacrylamide gel (SDS-PAGE) and then transferred electrophoretically onto polyvinylidene difluoride (PVDF) membranes (Millipore, Bedford, MA, USA). The blotted membranes were blocked with $5 \%$ non-fat dry milk (w/v) in Tris-buffered saline with $0.1 \%$ Tween-20 (TBS-T) and then incubated at $4{ }^{\circ} \mathrm{C}$ overnight with monoclonal antibodies against manganese superoxide dismutase (MnSOD), copper-zinc SOD (CuZnSOD), ERK, phospho-ERK, p38, phospho-p38 (Millipore), JNK, and phospho-JNK (Cell Signaling Technology, Boston, MA, USA), respectively. After six rinses with TBS-T at 5 min intervals, the membranes were incubated for $45 \mathrm{~min}$ with horseradish peroxidase-labeled goat anti-rabbit IgG. Protein bands were then visualized with an ECL plus chemiluminescence kit (Millipore). Immunoblotting with anti- $\beta$-actin antibody was used as an internal control to confirm equivalent protein loading. The obtained protein bands were scanned and quantified with the Gel pro 3.0 software.

Statistical analysis. Quantitative data are presented as mean \pm SD. Statistical analysis was adequately performed by

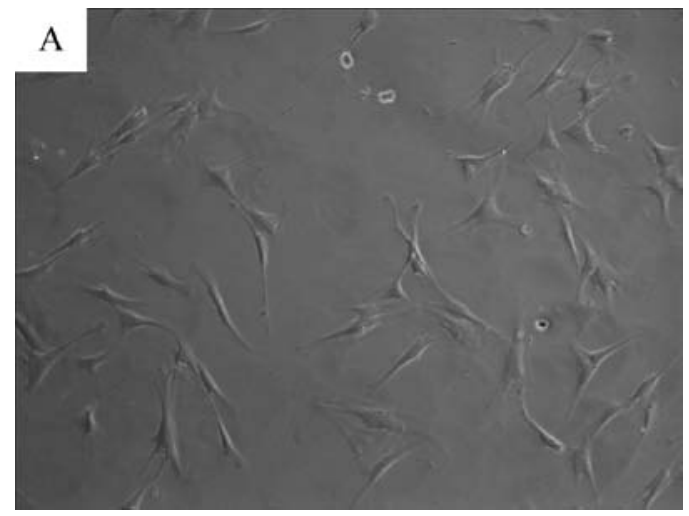

B
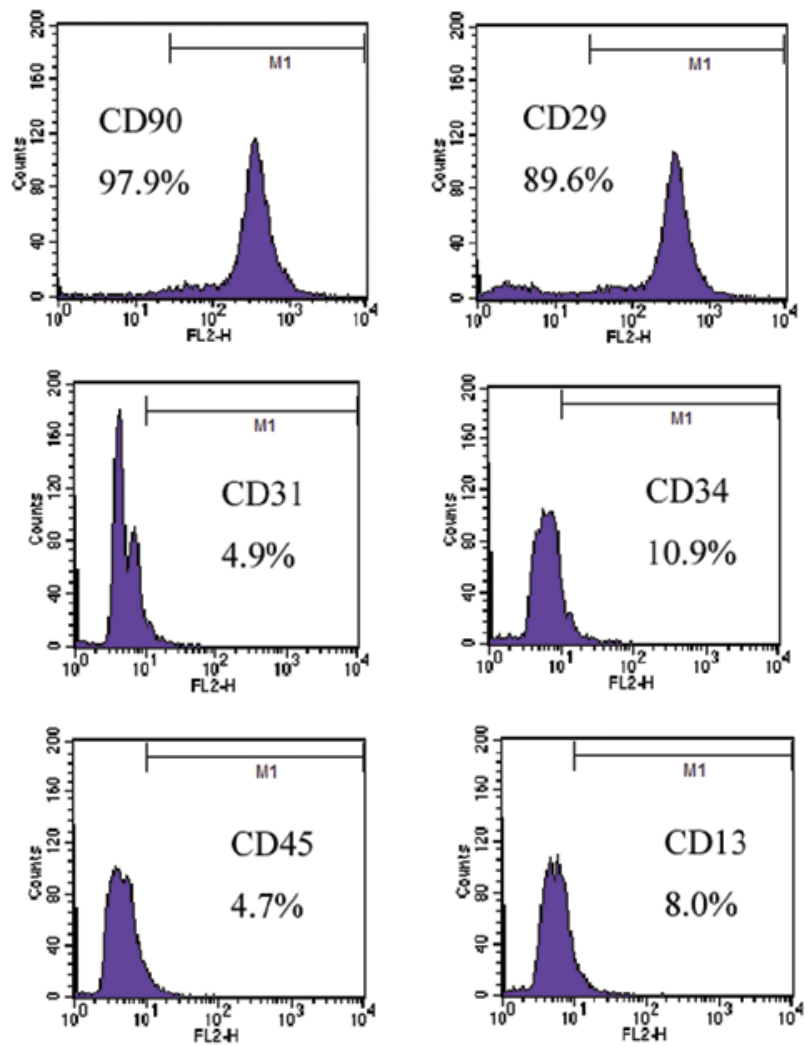

Figure 1. Flow cytometric analysis of rat ADMSCs. (A) Spindle-shaped morphological feature of the stem cells (magnification, x100). (B) Flow cytometric analysis of the second-passage ADMSCs showed that CD29+ and CD90+ cells were the highest population of stem cells.

ANOVA followed by Bonferroni multiple-comparison post hoc test using the SPSS software version 16.0 (SPSS, Inc., Chicago, IL, USA). A probability value $<0.05$ was considered to be statistically significant.

\section{Results}

Surface marker profiling of the isolated ADMSCs. Rat ADMSCs were isolated from the inguinal subcutaneous adipose tissue by their adherence to the plate. After grown for two passages in culture, contaminating hematopoietic cells were depleted during passaging, and ADMSCs were morphologically defined by a homogeneous population of 
Table I. The plasma biochemical parameters in the four groups of rats at 12 weeks.

\begin{tabular}{lrrrr}
\hline & \multicolumn{3}{c}{ Groups } \\
\cline { 2 - 5 } Parameters & Non-diabetic & \multicolumn{1}{c}{ ADMSC } & Vehicle & Diabetic \\
\hline Glucose $(\mathrm{mmol} / \mathrm{l})$ & $5.02 \pm 0.50$ & $14.27 \pm 2.10^{\mathrm{a}}$ & $29.12 \pm 3.30^{\mathrm{b}}$ & $28.86 \pm 1.43$ \\
Insulin $(\mathrm{mIU} / \mathrm{l})$ & $22.72 \pm 2.64$ & $16.69 \pm 1.16^{\mathrm{a}}$ & $11.27 \pm 1.40^{\mathrm{b}}$ & $10.94 \pm 1.17$ \\
Cholesterol $(\mathrm{mmol} / \mathrm{l})$ & $1.13 \pm 0.28$ & $1.23 \pm 0.15^{\mathrm{a}}$ & $1.82 \pm 0.26^{\mathrm{b}}$ & $2.01 \pm 0.28$ \\
Triglycerides $(\mathrm{mmol} / \mathrm{l})$ & $0.89 \pm 0.17$ & $1.25 \pm 0.16^{\mathrm{a}}$ & $1.80 \pm 0.18^{\mathrm{b}}$ & $1.74 \pm 0.14$ \\
Urea nitrogen $(\mathrm{mmol} / \mathrm{l})$ & $7.11 \pm 1.16$ & $13.14 \pm 2.69^{\mathrm{a}}$ & $18.34 \pm 0.92^{\mathrm{b}}$ & $18.77 \pm 0.77$ \\
Creatinine $(\mu \mathrm{mmol} / \mathrm{l})$ & $56.22 \pm 6.84$ & $73.53 \pm 3.35^{\mathrm{a}}$ & $93.21 \pm 5.58^{\mathrm{b}}$ & $94.67 \pm 8.45$ \\
\hline
\end{tabular}

Values are expressed as means $\pm \mathrm{SD}(\mathrm{n}=8) .{ }^{\mathrm{a}} \mathrm{P}<0.01 \mathrm{vs}$. the vehicle group; ${ }^{\mathrm{b}} \mathrm{P}<0.01$ vs. the non-diabetic group.
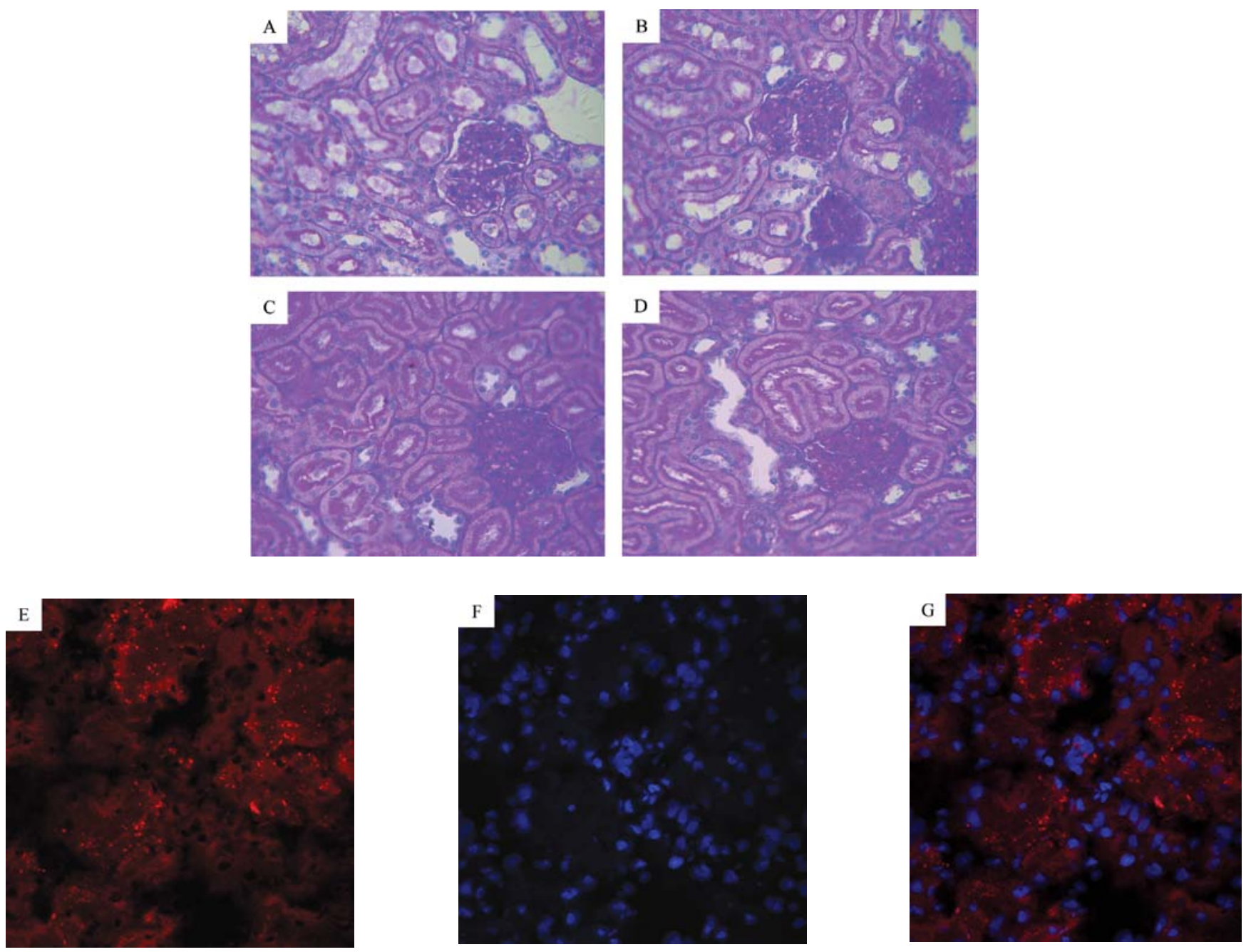

Figure 2. Effects of ADMSC transplantation on mesangial expansion in diabetic nephropathy. Photomicrographs of rat glomeruli sections of the (A) non-diabetic, (B) ADMSC, (C) vehicle and (D) diabetic groups are represented at magnification, $\mathrm{x} 400$ from periodic acid-Schiff-stained kidney. (E-G) Identification of CM-Dil-stained ADMSCs (red fluorescence) in the renal tissues of rats from the ADMSC group. (E) CM-Dil staining; (F) DAPI staining; (G) merged results. Original magnification, $\mathrm{x} 400$.

spindle-shaped adherent appearance (Fig. 1A). In addition, flow cytometry analysis showed that ADMSCs expressed CD29 and CD90, but exhibited negligible expression of CD34, CD31, CD45 and CD13 (Fig. 1B), with findings compatible with previous studies $(22,23)$.
Autologous transplantation of ADMSCs improves metabolic disorder in an STZ-induced diabetic model. The effectiveness of autologous transplantation of ADMSCs in alleviating metabolic disorder was assessed in the STZ-induced diabetic model, and common metabolic disorder parameters associated 
A

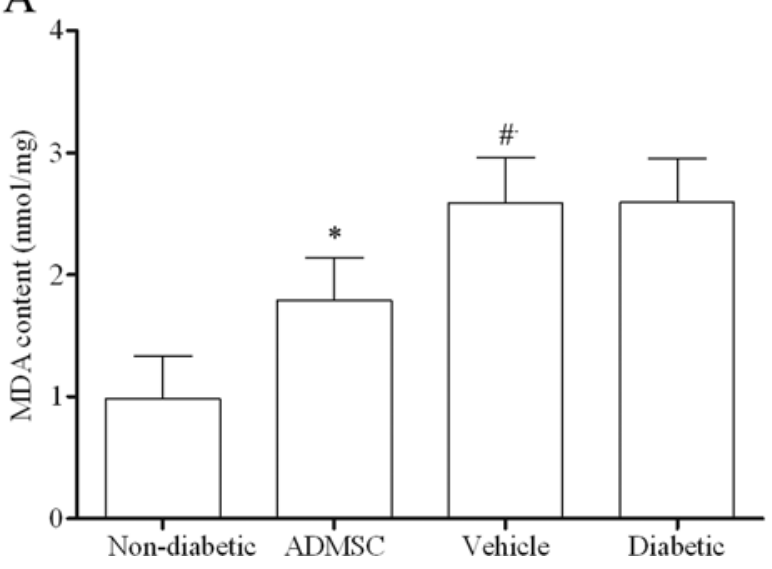

$\mathrm{C}$

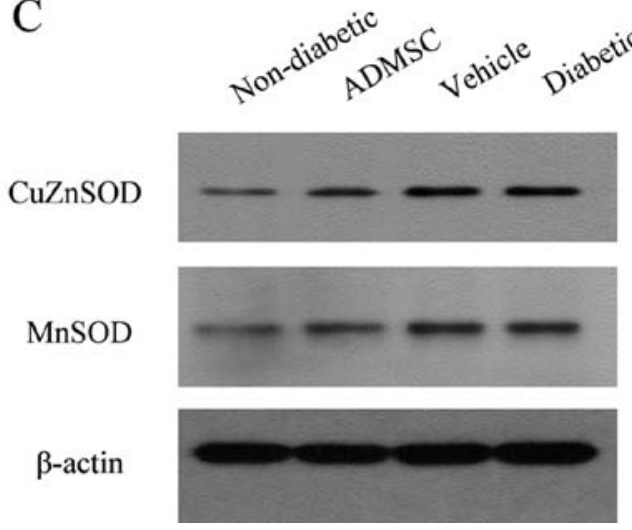

B
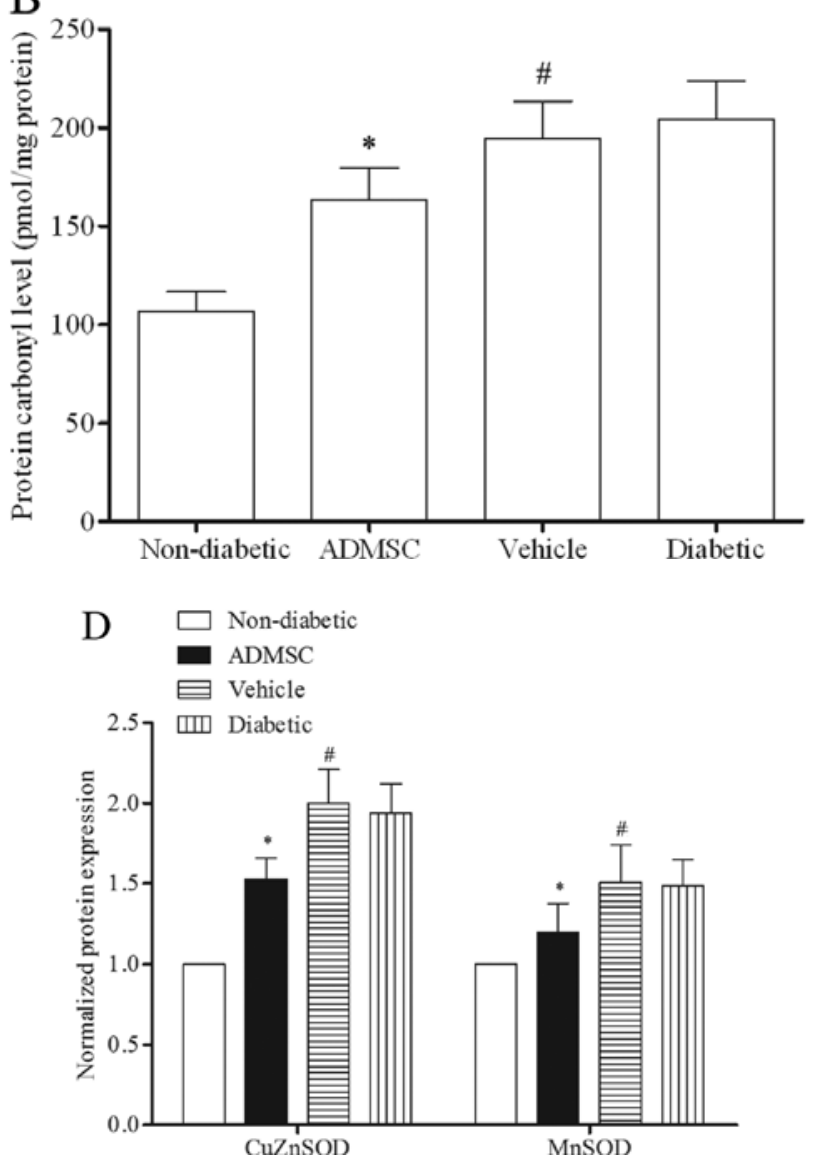

Figure 3. Effects of ADMSC transplantation on MDA content, protein carbonyl level and MnSOD and CuZnSOD protein expression in renal tissues of STZinduced diabetic rats. (A) The MDA content was determined using the modified thiobarbituric acid reactive substances method. (B) Protein carbonyl level was evaluated by 2,4-dinitrophenylhydrazine method. (C) Protein expressions of MnSOD and CuZnSOD were determined by western blot analysis. Representative blots are shown and protein size is expressed in $\mathrm{kDa}$. (D) Quantitative data of protein levels were expressed as the intensity ratio of target proteins to $\beta$-actin. Data represent the mean $\pm \mathrm{SD}$. ( $\mathrm{n}=8$ /group). ${ }^{*} \mathrm{P}<0.01$ vs. the vehicle group; ${ }^{*} \mathrm{P}<0.01$ vs. the non-diabetic group.

to diabetes were measured. In response to STZ, rats showed significantly increased plasma biochemical parameters, including glucose, cholesterol, triglycerides, urea nitrogen and creatinine. Importantly, autologous ADMSC implantation significantly alleviated all indices of metabolic dysfunction (Table I). Additionally, STZ injection decreased insulin level significantly, which was also attenuated by autologous transplantation of ADMSCs, suggesting that the protective effects of ADMSCs implantation may be associated with circulating insulin. Taken together, these data indicate that autologous transplantation of ADMSCs significantly attenuated metabolic disorder induced by STZ and that this protective effect is dependent on circulating insulin levels.

Autologous transplantation of ADMSCs alleviates renal damage induced by STZ. To further investigate the therapeutic effects of autologous ADMSCs transplantation on the improvement of kidney performance in the STZ-induced diabetic model, pathological changes of the kidney were measured. As shown in Fig. 2A, the renal tissue stained by Periodic acid and Schiff's solution appeared normal glomeruli in the renal cortex of non-diabetic controls. In contrast, it was observed that diabetes-induced histopathological changes in the renal tissues, including the expansion of mesangial matrix and thickening of glomerular basement (Fig. 2C and D). After autologous ADMSCs transplantation, enlargement of mesangia in glomeruli was significantly restored in the diabetes-affected renal tissues (Fig. 2B).

To determine whether transplanted ADMSCs were really homing to the renal tissues, double stain of CM-Dil and 4', 6-diamidino-2-phenylindole (DAPI) was performed. As expected, numerous CM-Dil-positive cells were identified in renal parenchyma of rats in the ADMSC group, indicating that ADMSCs was capable of migrating to renal tissues in the STZ-induced diabetic model (Fig. 2E-G).

ADMSCs transplantation ameliorates oxidative stress in the STZ-induced type 1 diabetic model. To determine whether ADMSC transplantation exerts its renoprotective effects on STZ-induced renal damage by inhibiting oxidative stress, the indicators of oxidative stress were measured. As shown in Fig. 3A and B, diabetes significantly increased renal content of MDA and protein carbonyl, markers of oxidative stress, in comparison to the non-diabetic normal group. Treatment diabetic rats with ADMSC transplantation significantly inhibited the increases of MDA and carbonyl protein in comparison to the vehicle treated and untreated diabetic animals. In addition, western blot analysis demonstrated 


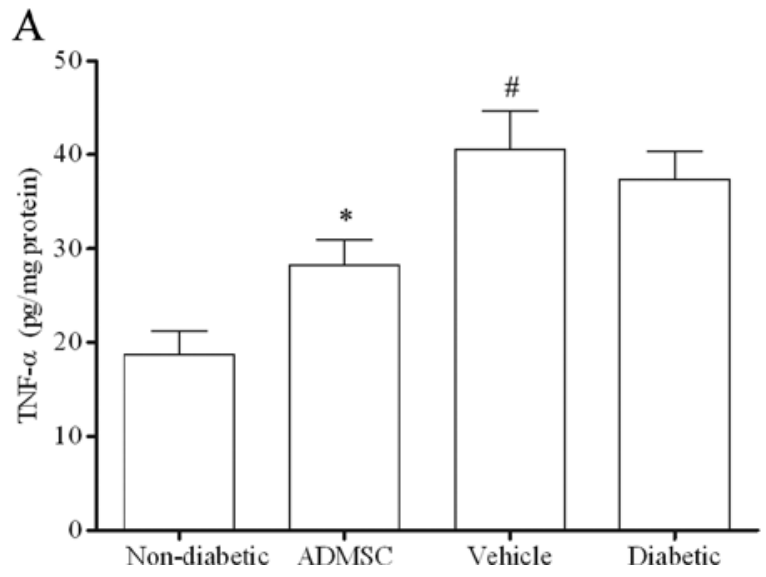

$\mathrm{B}$
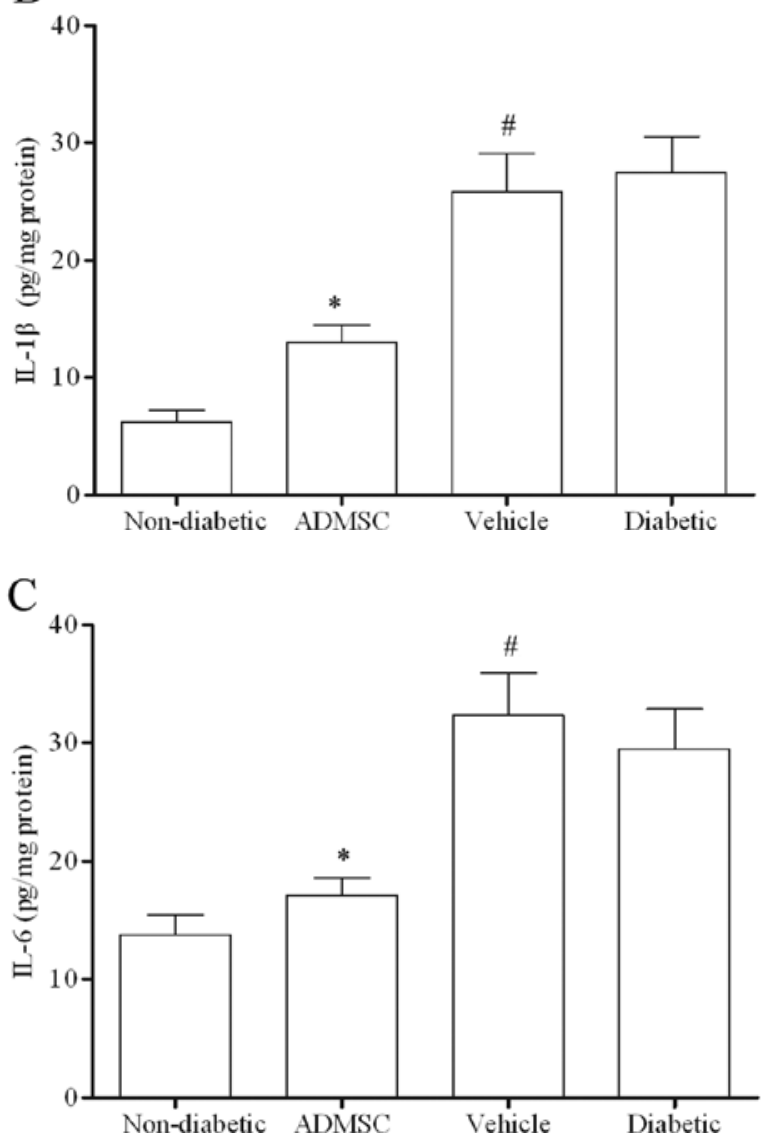

Figure 4. Effects of ADMSC transplantation on TNF- $\alpha$, IL-1 $\beta$ and IL-6 levels in the renal tissues of STZ-induced diabetic rats. Samples were processed to measure (A) TNF- $\alpha$, (B) IL-1 $\beta$ and (C) IL- 6 using a competitive ELISA Results are expressed as the mean $\pm \mathrm{SD}$ ( $\mathrm{n}=8$ /group). ${ }^{*} \mathrm{P}<0.01$ vs. the vehicle group; ${ }^{~} \mathrm{P}<0.01$ vs. the non-diabetic group.

significant increases of MnSOD and CuZn-SOD expression in the kidneys of diabetic animals and these increases of MnSOD and $\mathrm{CuZn-SOD}$ expression were remarkably attenuated by ADMSC transplantation in diabetic rats (Fig. 3C and D). These findings suggest that ADMSC transplantation reduced oxidative stress in diabetic animals.

ADMSCs transplantation decreases TNF- $\alpha, I L-1 \beta$ and $I L-6$ cytokine levels in the diabetic kidneys. To investigate the role of pro-inflammatory mediators on ADMSC-mediated
A

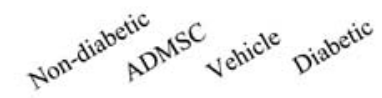

p-ERK

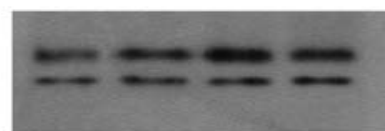

$43 \mathrm{kDa}$

ERK

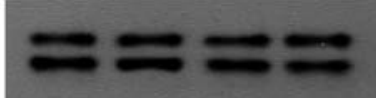

$43 \mathrm{kDa}$

p-JNK

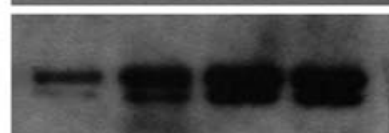

$50 \mathrm{kDa}$

JNK

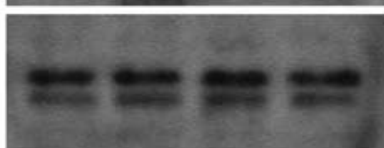

$50 \mathrm{kDa}$

p-p38

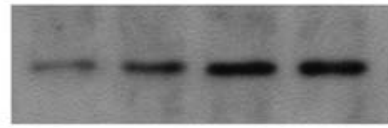

$38 \mathrm{kDa}$

p38

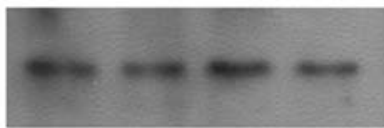

$38 \mathrm{kDa}$

$\beta$-actin

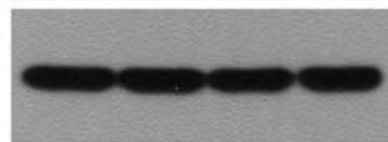

$43 \mathrm{kDa}$

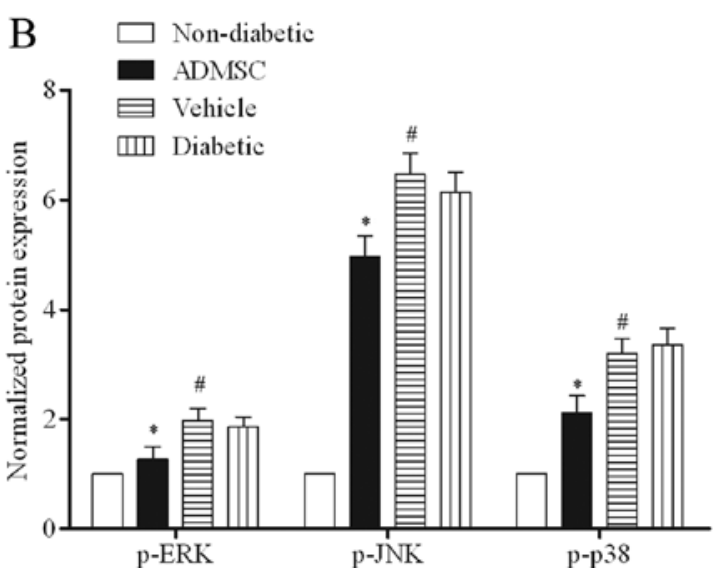

Figure 5. Effects of ADMSC transplantation on ERK, p38 and JNK phosphorylation and total protein expressions in the renal tissues of STZ-induced diabetic rats. Equal amounts of protein were resolved on SDS-PAGE and blotted with ERK, phospho- ERK , p38, phospho-p38, JNK and phospho-JNK antibodies. (A) Representative blots are shown and protein size is expressed in $\mathrm{kDa}$. (B) Quantitative data of protein levels were expressed as the intensity ratio of target proteins to $\beta$-actin. Columns represent the mean $\pm \mathrm{SD}$ ( $\mathrm{n}=8 /$ group). ${ }^{*} \mathrm{P}<0.01$ vs. the vehicle group; ${ }^{\text {}} \mathrm{P}<0.01$ vs. the non-diabetic group.

renoprotective effect, renal concentrations of TNF- $\alpha$, IL-1 $\beta$ and IL- 6 were determined by ELISA. As shown in Fig. 4, (A) TNF- $\alpha$, (B) IL-1 $\beta$ and (C) IL-6 levels were induced in vehicle treated animals by $\sim 120,300$ and $100 \%$, respectively, as compared to non-diabetic controls. ADMSC transplantation significantly inhibited the diabetes-mediated induction of TNF- $\alpha$, IL-1 $\beta$ and IL- 6 , suggesting that ADMSC implantation exerts its renoprotective effect via the inhibition of pro-inflammatory cytokines. 
ADMSC transplantation inhibits STZ-induced activation of the MAPK signaling pathway. It is well known that the expression of pro-inflammatory mediators is regulated by the activation of the MAPK pathway. Therefore, we assayed the effect of ADMSC implantation on the activation of the MAPK signaling pathways by examining the phosphorylation of p38, JNK and ERK by western blot analysis. As shown in Fig. 5, STZ injection resulted in significantly increased phosphorylation of all three MAPKs and these increases were remarkably attenuated by ADMSC implantation in diabetic rats, in comparison to the vehicle treated and untreated diabetic animals. These data suggest that ADMSC implantation suppresses pro-inflammatory cytokines through inhibiting the MAPK signaling pathway.

\section{Discussion}

In the present study, we demonstrated that autologous ADMSC implantation significantly decreased plasma glucose, cholesterol, triglycerides, urea nitrogen and creatinine and increased serum insulin level to moderate extents in the STZ-diabetic rats. Additionally, ADMSC implantation effectively alleviated oxidative stress and prevented pro-inflammatory cytokines and the MAPK signaling pathway in renal tissues of diabetic rats, suggesting the possible mechanisms by which ADMSC implantation exerts its renoprotective effects in the diabetic rats. To our knowledge, this is the first report demonstrating the potential therapeutic effects of autologous ADMSCs implantation on diabetic nephropathy in an STZ-induced diabetic rat model.

Because of their immunomodulatory ability, capacity for self-renewal and differentiation into mesodermal tissues, stem cells have been considered to be attractive therapeutic agents for a number of diseases including complications of diabetes mellitus. Nowadays, several candidates of stem cells, including embryonic stem cells, BMMSCs and peripheral blood-derived stem cells have been commonly investigated for their feasibility and safety in the treatment of diabetes mellitus and its complications in both clinical observational studies and animal models (24). However, the application of these stem cells for diabetic patients is frequently hampered by many limitations, including ethical problems with using embryonic stem cells, difficulties in differentiation lineage restriction and identification as well as limitation of number and functional integrity in peripheral blood-revived stem cells and BMMSCs $(25,26)$. The most important finding in the present study is that numerous CM-Dil-labeled ADMSCs were identified in renal parenchyma of diabetic rats at 12 weeks after induction of diabetes. Moreover, transplantation of ADMSCs significantly ameliorated renal damage induced by diabetes, as evidenced by histopathological results. These findings, in addition to support our original hypothesis, may provide a good cell source, i.e. ADMSCs, for transplantation. The administration of ADMSCs through the systemic venous route has also been validated in this study. Our results, therefore, offer a potential clinical avenue for the future use of ADMSCs in diabetes mellitus.

Hyperglycemia has been shown to promote oxidative stress in nephritic tissues, eventually leading to renal injury in diabetes $(27,28)$. Additionally, several clinical and animal studies have indicated that inflammatory cytokines play an important role in the development and progression of diabetic nephropathy $(29,30)$. It was demonstrated that diabetes increased proinflammatory renal production, including TNF- $\alpha$, IL- $1 \beta$ and IL-6 in the circulating, and urinary excretion (31-33). Another important finding in the current study is that the levels of MDA and protein carbonyl group and protein expression of MnSOD and $\mathrm{CuZn-SOD}$ in renal tissues as well as TNF- $\alpha$, IL-1 $\beta$ and IL-6 in plasma were remarkably increased in the vehicle and diabetic groups compared to non-diabetic rats. In this way, our findings reinforce those of previous studies. Of importance is that, as compared to diabetic animals with vehicle treatment, the levels of these inflammatory and oxidative biomarkers were significantly suppressed in animals following ADMSCs administration. There is accumulating evidence indicating that MSCs have distinctive immunomodulatory property that contributes to the downregulation of inflammatory reaction under diabetic condition $(34,35)$. Therefore, our findings are consistent with those of previous studies. Taken together, these data suggest that ADMSCs treatment exerts its renoprotective effects, at least in part, through inhibiting inflammatory reactions and oxidative stress in this experimental setting of STZ-induced diabetes.

p38 MAPK signaling pathway is essential for the regulation of many cellular processes, including inflammation, cell differentiation, cell growth and cell death (36). There is mounting evidence that supports the central role of the p38 MAPK signaling pathway in high glucose-induced cell damage. Recent studies have demonstrated that high levels of glucose can activate the p38 MAPK signaling pathway in renal cells and induce the phosphorylation of p38 MAPK, and high expression of the MAPK pathway mRNAs correlates with glomerular lesions in human diabetic nephropathy $(37,38)$. In the present study, we consistently found that all three MAPKs, p38, JNK and ERK, were activated in renal tissues of diabetic animals. However, these increases of phosphorylation were attenuated by ADMSC administration. It is well known that the expression of pro-inflammatory mediators is regulated by the activation of the MAPK pathway. Therefore, our results suggest that ADMSC administration may suppress inflammatory reactions through inhibiting the MAPK signaling pathway in kidneys of diabetic animals.

In conclusion, the current study demonstrated that autologous transplantation of ADMSCs protects against diabetic nephropathy by restoring the biochemical alterations as well as inhibition of oxidative stress, pro-inflammatory gene expression levels and the p38 MAPK signaling pathway, suggesting a potential clinical use of ADMSCs to prevent the onset and progression of diabetic nephropathy.

\section{Acknowledgements}

This study was supported by a grant from the Scientific Research Startup Foundation for Youths of Liaoning Medical University (Grant no. Y2010Z007).

\section{References}

1. Dronavalli S, Duka I and Bakris GL: The pathogenesis of diabetic nephropathy. Nat Clin Pract Endocrinol Metab 4: 444-452, 2008. 
2. Kanwar YS, Sun L, Xie P, Liu FY and Chen S: A glimpse of various pathogenetic mechanisms of diabetic nephropathy. Annu Rev Pathol 6: 395-423, 2011.

3. Kanwar YS, Wada J, Sun L, et al: Diabetic nephropathy: mechanisms of renal disease progression. Exp Biol Med (Maywood) 233: 4-11, 2008.

4. Soldatos G and Cooper ME: Diabetic nephropathy: important pathophysiologic mechanisms. Diabetes Res Clin Pract 82: S75-S79, 2008

5. Gross JL, de Azevedo MJ, Silveiro SP, Canani LH, Caramori ML and Zelmanovitz T: Diabetic nephropathy: diagnosis, prevention, and treatment. Diabetes Care 28: 164-176, 2005.

6. Burney BO, Kalaitzidis RG and Bakris GL: Novel therapies of diabetic nephropathy. Curr Opin Nephrol Hypertens 18: 107-111, 2009.

7. Pittenger MF, Mackay AM, Beck SC, et al: Multilineage potential of adult human mesenchymal stem cells. Science 284 143-147, 1999.

8. Liu ZJ, Zhuge Y and Velazquez OC: Trafficking and differentiation of mesenchymal stem cells. J Cell Biochem 106: 984-991, 2009.

9. Ma T: Mesenchymal stem cells: From bench to bedside. World J Stem Cells 2: 13-17, 2010.

10. Locke M, Windsor J and Dunbar PR: Human adipose-derived stem cells: isolation, characterization and applications in surgery. ANZ J Surg 79: 235-244, 2009.

11. Dekel B, Shezen E, Even-Tov-Friedman S, et al: Transplantation of human hematopoietic stem cells into ischemic and growing kidneys suggests a role in vasculogenesis but not tubulogenesis. Stem Cells 24: 1185-1193, 2006.

12. Togel F, Weiss K, Yang Y, Hu Z, Zhang P and Westenfelder C: Vasculotropic, paracrine actions of infused mesenchymal stem cells are important to the recovery from acute kidney injury. Am J Physiol Renal Physiol 292: F1626-F1635, 2007.

13. Bi B, Schmitt R, Israilova M, Nishio H and Cantley LG: Stromal cells protect against acute tubular injury via an endocrine effect. J Am Soc Nephrol 18: 2486-2496, 2007.

14. da Silva LB, Palma PV, Cury PM and Bueno V: Evaluation of stem cell administration in a model of kidney ischemia-reperfusion injury. Int Immunopharmacol 7: 1609-1616, 2007.

15. Li B, Cohen A, Hudson TE, Motlagh D, Amrani DL and Duffield JS: Mobilized human hematopoietic stem/progenitor cells promote kidney repair after ischemia/reperfusion injury. Circulation 121: 2211-2220, 2010.

16. Liu M and Han ZC: Mesenchymal stem cells: biology and clinical potential in type 1 diabetes therapy. J Cell Mol Med 12 1155-1168, 2008.

17. Vanikar AV, Dave SD, Thakkar UG and Trivedi HL: Cotransplantation of adipose tissue-derived insulin-secreting mesenchymal stem cells and hematopoietic stem cells: a novel therapy for insulin-dependent diabetes mellitus. Stem Cells Int 2010: 582382, 2010.

18. Yang Z, Li K, Yan X, Dong F and Zhao C: Amelioration of diabetic retinopathy by engrafted human adipose-derived mesenchymal stem cells in streptozotocin diabetic rats. Graefes Arch Clin Exp Ophthalmol 248: 1415-1422, 2010.

19. Zhang H, Qiu X, Shindel AW, et al: Adipose Tissue-Derived Stem Cells Ameliorate Diabetic Bladder Dysfunction in a Type II Diabetic Rat Model. Stem Cells Dev: October 18, 2011 (Epub ahead of print).
20. Zuk PA, Zhu M, Ashjian P, et al: Human adipose tissue is a source of multipotent stem cells. Mol Biol Cell 13: 4279-4295, 2002.

21. Calabrese V, Mancuso C, Sapienza M, et al: Oxidative stress and cellular stress response in diabetic nephropathy. Cell Stress Chaperones 12: 299-306, 2007.

22. Strem BM, Hicok KC, Zhu M, et al: Multipotential differentiation of adipose tissue-derived stem cells. Keio J Med 54: 132-141, 2005.

23. Wang L, Deng J, Tian W, et al: Adipose-derived stem cells are an effective cell candidate for treatment of heart failure: an MR imaging study of rat hearts. Am J Physiol Heart Circ Physiol 297: H1020-H1031, 2009.

24. Bernardi S, Severini GM, Zauli G and Secchiero P: Cell-based therapies for diabetic complications. Exp Diabetes Res 2012: 872504, 2012.

25. Mitjavila-Garcia MT, Simonin C and Peschanski M: Embryonic stem cells: meeting the needs for cell therapy. Adv Drug Deliv Rev 57: 1935-1943, 2005.

26. Volarevic V, Arsenijevic N, Lukic ML and Stojkovic M: Concise review: Mesenchymal stem cell treatment of the complications of diabetes mellitus. Stem Cells 29: 5-10, 2011.

27. Forbes JM, Coughlan MT and Cooper ME: Oxidative stress as a major culprit in kidney disease in diabetes. Diabetes 57: 1446-1454, 2008.

28. Yoh K, Hirayama A, Ishizaki K, et al: Hyperglycemia induces oxidative and nitrosative stress and increases renal functional impairment in Nrf2-deficient mice. Genes Cells 13: 1159-1170, 2008.

29. Navarro JF and Mora C: Role of inflammation in diabetic complications. Nephrol Dial Transplant 20: 2601-2604, 2005.

30. Wolkow PP, Niewczas MA, Perkins B, et al: Association of urinary inflammatory markers and renal decline in microalbuminuric type 1 diabetics. J Am Soc Nephrol 19: 789-797, 2008.

31. Matavelli LC, Huang J and Siragy HM: (Pro)renin receptor contributes to diabetic nephropathy by enhancing renal inflammation. Clin Exp Pharmacol Physiol 37: 277-282, 2010.

32. Huang $\mathbf{J}$ and Siragy HM: Glucose promotes the production of interleukine-1beta and cyclooxygenase- 2 in mesangial cells via enhanced (Pro)renin receptor expression. Endocrinology 150: 5557-5565, 2009.

33. Kaul K, Hodgkinson A, Tarr JM, Kohner EM and Chibber R: Is inflammation a common retinal-renal-nerve pathogenic link in diabetes? Curr Diabetes Rev 6: 294-303, 2010.

34. Abdi R, Fiorina P, Adra CN, Atkinson M and Sayegh MH: Immunomodulation by mesenchymal stem cells: a potential therapeutic strategy for type 1 diabetes. Diabetes 57: 1759-1767, 2008.

35. Volarevic V, Arsenijevic N, Lukic ML and Stojkovic M: Mesenchymal stem cell treatment of complications of diabetes mellitus. Stem Cells 29: 5-10, 2011.

36. Ono K and Han J: The p38 signal transduction pathway: activation and function. Cell Signal 12: 1-13, 2000.

37. Wilmer WA, Dixon CL and Hebert C: Chronic exposure of human mesangial cells to high glucose environments activates the p38 MAPK pathway. Kidney Int 60: 858-871, 2001.

38. Toyoda M, Suzuki D, Honma M, et al: High expression of PKC-MAPK pathway mRNAs correlates with glomerular lesions in human diabetic nephropathy. Kidney Int 66: 1107-1114, 2004. 